\title{
APPAREIL POUR PRÉLĖVEMENT ET TESTS BIOLOGIQUES IN SITU
}

\author{
Réalisation Technique par M. Michel SAVARY *
}

\section{1 - INTERET ET NECESSITE DES TESTS BIOLOGIQUES IN SITU}

-- L'apparition de matières polluantes nouvelles pose des problèmes d'analyse chimique difficiles : il existe un certain décalage entre l'apparition d'une substance dans les eaux et la mise au point de sa méthode d'analyse à l'état de résidu.

D'autre part, certaines substances toxiques bien connues sont susceptibles d'évoluer pendant le temps de transport des échantillons prélevés à la source même de la pollution.

- Il s'est donc avéré nécessaire de réaliser directement sur les effluents, des tests permettant de déterminer leur toxicité immédiate ou à court terme.

- La réalisation de ces tests permet également de limiter le nombre de prélèvements destinés au laboratoire d'analyses; la prise d'échantillon (notamment dans le cas d'effluents de qualité variable) étant effectuée à bon escient.

- Les résultats de ces opérations jouent un rôle déterminant dans les décisions judiciaires en ce qui concerne l'application de l'article 434-1 du Code Rural.

* Garde-Chef du Conseil Supérieur de la Pêche, mis à la disposition de la Division Qualité des Eaux, Pêche et Pisciculture. 


\section{2 - MISE AU POINT DE L'APPAREILLAGE}

\subsection{Prélèvement de l'eau à étudier}

- Il est essentiel de réaliser le prélèvement d'un effluent avant son mélange avec le cours d'eau récepteur : dans le cas d'un déversement au-dessous du niveau du cours d'eau, le prélèvement direct, à l'aide d'un récipient, est aléatoire sinon impossible.

D'autre part, il est parfois nécessaire d'opérer des prélèvements à l'intérieur même des conduites enterrées, par l'intermédiaire des orifices de visite.

- Ces impératifs ont conduit à la mise au point d'un appareillage simple et autonome, procédant par pompage de l'eau à étudier.

\subsection{Localisation des effluents}

Dans le cas d'effluents débouchant sous le niveau du cours d'eau, le système de pompage permet de localiser avec précision le point de déversement, grâce à la détermination immédiate de certains paramètres (température, $\mathrm{pH}$, conductivité) effectuée en continu sur l'eau pompée.

- On notera que la recherche de la plus forte concentration dans une nappe polluée descendant le cours d'une rivière peut être effectuée par la même méthode.

\subsection{Tests biologiques}

Les tests de toxicité sont, en général, réalisés sur des poissons; toutefois, l'utilisation d'invertébrés aquatiques peut être envisagée : l'emploi simultané ou séparé de poissons et d'invertébrés étant susceptible de permettre une meilleure compréhension de certains effets de la pollution.

- Dans la pratique, les organismes servant au test sont placés dans une enceinte balayée en continu par l'eau pompée dans l'effluent.

- Un système d'aération de l'eau prélevée est installé dans le circuit de pompage, il est destiné à aérer certaines eaux résiduaires qui sont seulement désoxygénées (eaux de refroidissement, de condensation) sans pour autant être toxiques, ce qui permet de lever toute ambiguïté sur la toxicité des effluents testés.

\section{3 - DESCRIPTION DE L'APPAREIL}

- Le pompage est assuré par un groupe électrique de faible puissance.

- L'aspiration s'effectue par l'intermédiaire d'un tube souple muni à son extrémité d'une crépine lestée, avec possibilité d'intercaler un tube rigide entre la crépine et le tube souple, ce qui permet un meilleur guidage lors des recherches.

- L'eau pompée traverse un bac muni d'un trop-plein; ce bac reçoit les organismes destinés au test biologique, il est muni d'un couvercle transparent.

- Le système d'aération est disposé à la sortie de la pompe.

- Une bonne partie de l'appareillage peut être réalisée sur place en s'inspirant de la présente description selon les disponibilités locales.

- Les cotes données ne sont pas impératives, notamment en ce qui concerne les dimensions du bac. 
- L'alimentation du dispositif (12 volts continu) peut être réalisée à partir d'un groupe électrogène portatif ou d'une batterie d'automobile. Dans ce dernier cas, il y a intérêt à utiliser une batterie annexe plutôt que celle du véhicule utilisé pour se rendre sur le terrain.

La consommation est de 7 à 8 ampères sous 12 volts. La durée de pompage disponible dépend de la capacité de l'accumulateur employé (environ 4 à 5 heures pour une batterie de $50 \mathrm{Ah}$ ). L'autonomie peut être accrue en renonçant au pompage en continu et en procédant au renouvellement du volume du bac de façon périodique ou en utilisant une pompe présentant une consommation inférieure, le débit obtenu avec le modèle actuellement en service pouvant être réduit de moitié sans inconvénient. Les recherches se poursuivent dans ce sens.

\section{4 - CARACTERISTIQUES D'UN MODELE EN SERVICE}

- Bac :

- Réalisé en PVC de $4 \mathrm{~mm}$ d'épaisseur.

- Dimensions en mm : $450 \times 150 \times 150$.

- Hauteur d'eau, appareil en service : $100 \mathrm{~mm}$ environ.

- Deux grilles (amont et aval) en PVC perforé - trous de $\varnothing 8 \mathrm{~mm}$.

- Pompe :

- Minipompe JABSCO - modèle 7600 (12 volts) distribuée par "Pompes GUINARD • : avenue de Fouilleuse - 92 SAINT-CLOUD.

\section{- Aération :}

- Trompe à eau du commerce dont la voie a été augmentée jusqu'à $7 \mathrm{~mm}$.

\section{- Tubes d'aspiration :}

- Tube rigide en dural $\varnothing$ intérieur : $16 \mathrm{~mm}$.

- Tube souple (tuyau d'arrosage) $\varnothing$ intérieur : $13 \mathrm{~mm}$.

\section{- Crépine :}

- Lestée à $1,5 \mathrm{~kg}$. Orifices à $150 \mathrm{~mm}$ de la base.

\section{- Autonomie :}

- 1 à 2 heures, sur la batterie d'une voiture. 


\section{5 - PLANS DE L'APPAREIL}
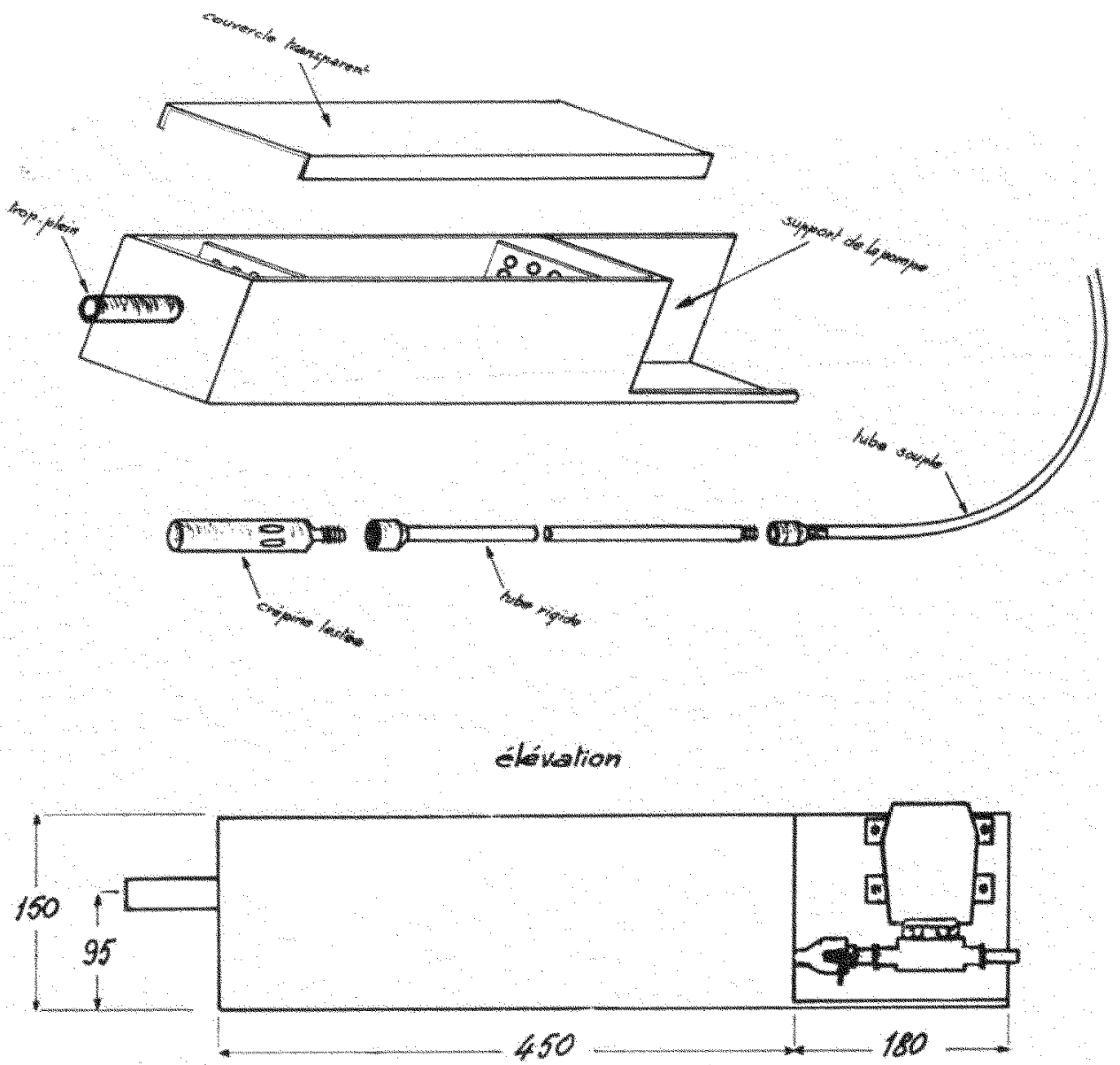

we plan

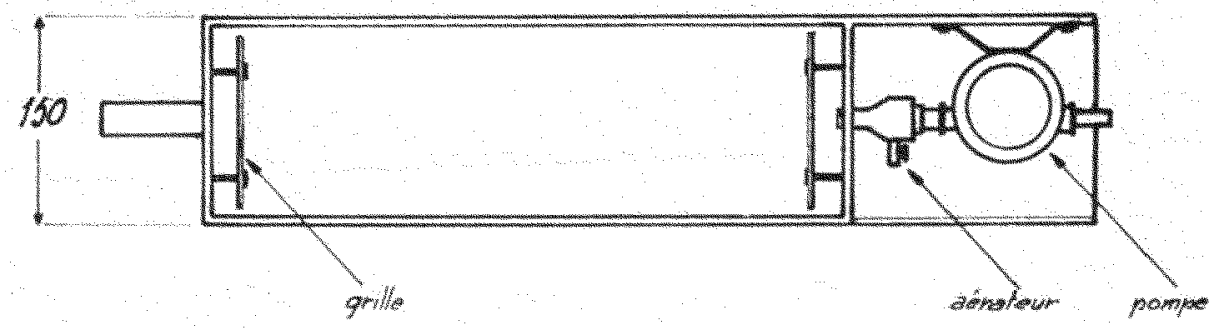

